\title{
QUALIDADE FISIOLÓGICA DE SEMENTES DE SORGO ATRAVÉS O TESTE DE ESTRESSE HÍDRICO
}

\author{
PHYSIOLOGICAL QUALITY OF GRAIN SORGHUM SEEDS \\ BY THE HIDRIC STRESS TEST
}

\author{
Salvador Barros Torres ${ }^{1}$
}

\section{RESUMO}

\begin{abstract}
Com o objetivo de estudar a eficiência do teste de estresse hídrico na avaliação da qualidade fisiológica de sementes de sorgo (Sorghum bicolor (L.) Moench), seis lotes de sementes básicas da cultivar IPA-1011, safra 1995, foram analisados pêlos testes de germinação, primeira contagem, de frio sem solo, envelhecimento acelerado, condutividade elétrica, emergência em campo e comprimento das plântulas sob estresse hídrico nos potenciais de $\mathrm{O},-0,3$. -0,6 e -0,9 MPa. Os trabalhos foram realizados no Laboratório de Análise de Sementes e no campo experimental da EMBRAPA-CPATSA, em Petrolina, PE. As determinações foram conduzidas com quatro repetiç̃es em delineamento experimental inteiramente casualizado. Os resultados indicaram que o teste de germinação sob estresse hídrico de -0,6MPa, pode ser usado para estimar o desempenho das sementes de sorgo em situações desfavoráveis de disponibilidade hídrica no solo.
\end{abstract}

Palavras-chave: Sorghum bicolor (L.) Moench, polietileno glicol, teste de vigor.

\section{SUMMARY}

This work was carried out with the objective of testing the efficiency of the water stress test of the evaluation of physiological quality of grain sorghum (Sorghum bicolor (L.) Moench) seeds. Six lots of basic seeds of the cultivar IPA-1011 were analyzed by the following tests: germination, first count, cold without soil test, accelerated aging, electrical conductivity, field emergency, and seedling length under hydric stress at $\mathrm{O}$, -
0.3, - 0.6 and -0.9MPa matric potentials. The trials were carried out at the Seed Laboratory at the experimental field of EMBRAPA-CPATSA, Petrolina - PE, in a completely randomized design with four replications. The results indicated that the germination test under water stress of -0,6MPa can be used to estimate the performance of grain sorghum seeds under unfavorable soil water conditions.

Key Words: Sorghum bicolor (L.) Moench, polyethylene glycol, test vigor.

\section{INTRODUÇÃO}

A qualidade fisiológica tem sido pesquisada pelo fato de as sementes estarem sujeitas a uma série de mudanças degenerativas associadas com a redução do vigor (ABDUL-BAKI \& ANDERSON, 1972). Segundo DELOUCHE \& BASKIN (1973), essa seqüência de mudanças tem início com a degeneração das membranas celulares, culminando com a perda do poder germinativo das sementes. Essa deterioração está diretamente relacionada ao vigor da semente que, por sua vez, tem sido avaliado através de testes, conforme recomendam INTERNATIONAL SEED TESTING ASSOCIATION - ISTA (1981) e ASSOCIATION OF OFFICIAL SEED ANALYSTS - AOSA (1983). Porém, em condições de campo, as

\footnotetext{
${ }^{1}$ Engenheiro Agrônomo, MsC., Pesquisador, EMPARN/EMBRAPA-CPATSA, Caixa Postal 23, 56300-000, Petrolina - PE.
} 
sementes poderão estar sujeitas a situações adversas de excesso ou déficit hídrico, obstrução mecânica imposta por compactação ou crostas do solo e por ataque de microorganismos e insetos (PERRY, 1981). Portanto, a porcentagem de emergência de plântulas, geralmente, é inferior a sugeridos pêlos testes de germinação, pelo fato deste ser operacionalizado em condições favoráveis (JOHNSON \& WAX, 1978 e YAKLICH \& KULIK, 1979). Em função disso, tem-se procurado um método de avaliação das sementes com sensibilidade suficiente para estimar com maior precisão a qualidade das sementes, como o teste de frio em milho, e o envelhecimento acelerado, em soja.

Outro teste citado pela AOSA (1983) é o estresse osmótico, cujo método fundamenta-se no princípio de que sementes mais vigorosas podem tolerar condições mais severas de déficit hídrico (HADAS, 1977). Segundo El-SHARKAWI \& SPRINGUEL (1977), a taxa de germinação e as emergências tanto da radícula como da plúmula são reduzidas, em condições de déficit hídrico. De maneira geral, a redução progressiva do potencial hídrico do substrato provoca decréscimo no comprimento das plântulas e na porcentagem de germinação (EMMERICH \& HARDEGREE, 1990 e GERMU \& NAYLOR, 1991). Em termos gerais, menor qualidade fisiológica tem sido associada aos piores desempenhos nessa condição (PARMAR \& MOORE, 1968 e MATTHEWS \& POWELL, 1986).

O objetivo do presente trabalho foi o de estudar a eficiência do teste de déficit hídrico, também denominado de teste estresse osmótico, na avaliação da qualidade fisiológica de sementes de sorgo (Sorghum bicolor (L.) Moench).

\section{MATERIAIS E MÉTODOS}

O trabalho foi desenvolvido no Laboratório de Analise de Sementes e no campo experimental do CPATSA-EMBRAPA, em Petrolina, PE, no período de novembro de 1995 a março de 1996. Foram utilizados seis lotes de sementes básicas de sorgo, cultivar IPA-1011, safra 1994/95, beneficiadas e não tratadas, provenientes do Serviço de Produção de Sementes Básicas-SPSBEMBRAPA, em Petrolina, PE.

Durante a fase experimental, as sementes permaneceram armazenadas em sacos de papel sob condições controladas $\left(10^{\circ} \mathrm{C}\right.$ e $35 \%$ de umidade relativa do ar). Para avaliar a qualidade fisiológica, as sementes foram submetidas aos seguintes testes: a) Teste de germinação - este teste foi conduzido a $25^{\circ} \mathrm{C}$ em rolos de papel toalha, com 4 repetições de 50 sementes, seguindo os procedimentos previstos nas Regras para Análise de Sementes (BRASIL, 1992). b) Primeira contagem de germinação efetuado conjuntamente com o teste de germinação, determinando-se a porcentagem de plântulas normais no quarto dia após a sua instalação, conforme prescrições das Regras para Análise de Sementes (BRASIL, 1992). c) Condutividade elétrica - selecionaram-se e pesaram-se 4 repetições de 25 sementes para cada lote; em seguida, foram imersas em $25 \mathrm{ml}$ de água destilada por 24 horas à temperatura constante de $25^{\circ} \mathrm{C}$. A leitura da condutividade elétrica da solução foi realizada em condutivímetro, sendo os resultados expressos em $\mu \mathrm{s} / \mathrm{cm} / \mathrm{g}$ de sementes (VIEIRA, 1994). d) Envelhecimento acelerado - utilizaram-se 200 sementes por lote, em 4 repetições de 50 sementes, distribuídas sobre bandejas de tela de alumínio, fixadas no interior de caixas de plástico, tipo gerbox, a $42^{\circ} \mathrm{C}$ e $100 \%$ de umidade relativa do ar, por um período de 96 horas (MARCOS FILHO, 1994). Decorrido este período, as sementes foram colocadas para germinar, seguindo-se as recomendações para o teste de germinação (BRASIL, 1992). A avaliação das plântulas foi realizada quatro dias após a semeadura, computando-se a porcentagem de plântulas normais. e) Teste de frio em rolo de papel sem solo - foi conduzido conforme recomendações de CÍCERO \& VIEIRA (1994), onde 4 repetições de 50 sementes foram distribuídas em rolos de papel umedecido em quantidade de água equivalente a 2,5 vezes o peso do papel. Após a semeadura, os rolos foram colocados no interior de sacos de plástico e vedados com fita adesiva, sendo mantidos em câmara regulada a $10^{\circ} \mathrm{C}$, durante 7 dias. Após este período, os rolos foram retirados dos sacos plásticos e transferidos para um germinador à temperatura constante de $25^{\circ} \mathrm{C}$, onde permaneceu por 4 dias; a seguir foi contado o número de plântulas normais (BRASIL, 1992). f) Emergência das plântulas em campo - o teste foi realizado com 4 repetições de 100 sementes por lote. As sementes, de cada repetição, foram semeadas, a $3 \mathrm{~cm}$ de profundidade, em sulcos com $5 \mathrm{~m}$ de comprimento e distanciados de $0,50 \mathrm{~m}$ entre si. As contagens, efetuadas aos 21 dias após a semeadura, determinaram a porcentagem de emergência das plântulas segundo os procedimentos descritos por NAKAGAWA (1994). g) Teste de estresse hídrico - foi conduzido em 4 situações de disponibilidade hídrica, utilizando-se os potenciais de $0,-0,3-0,6$ e $-0,9 \mathrm{MPa}$ obtidos em soluções aquosas de polietileno glicol (PEG 6.000). O 
cálculo, das quantidades de soluto, foi obtido com base na equação sugerida por MICHEL \& KAUFMANN (1973) e VILLELA $\boldsymbol{e t} \boldsymbol{a l}$. (1991). As soluções foram aplicadas sobre o papel substrato, em quantidade equivalente a 2,5 vezes o peso deste último.

Foram utilizadas quatro repetições de dez sementes por lote, semeadas eqüidistante sobre um traço horizontal demarcado no limite do terço superior da folha de papel. A seguir, após a superposição de mais uma folha de papel, formaram-se rolos, que foram embalados em sacos plásticos e levados para germinador regulado à temperatura constante de $25^{\circ} \mathrm{C}$, em ausência de luz, de maneira que as radículas ficassem-voltadas para baixo. O prazo de permanência das sementes em contato com as soluções foi de 10 dias. No final desse período, foram feitas medições do comprimento total das plântulas normais (mm) presentes na repetição, obtendo-se um índice, representado pela divisão do somatório dos comprimentos da população total de plântulas pelo número de sementes colocadas para germinar. Os dados obtidos em cada teste foram analisados separadamente através da análise da variância, e, conjuntamente, mediante análise de correlação. As médias obtidas foram comparadas pelo teste de Duncan a $1 \%$ de probabilidade.

\section{RESULTADOS E DISCUSSÃO}

Verificando os resultados da tabela 1 , constata-se que os testes não foram igualmente sensíveis para distinguir diferenças na qualidade fisiológica entre os lotes. Os testes de germinação, primeira contagem, frio em rolo de papel sem solo, envelhecimento acelerado e condutividade elétrico não se correlacionaram com o de emergência em campo (Tabela 2). Os resultados do teste de germinação foram apreciavelmente superiores ao de emergência em campo (Tabela 1). Isso se deve as condições ótimas que, geralmente, conduzem a superestimativa do desempenho das sementes em condições controladas (JOHNSON \& WAX, 1978 e YAKLICH \& KULIK, 1979).
Tabela 2 - Coeficiente de correlação simples (r) entre os resultados dos testes de laboratório e de emergência em campo para sementes de sorgo (Sorghum bicolor (L.) Moench).

\begin{tabular}{lc}
\hline Teste de laboratório x emergência em campo & $\mathrm{R}$ \\
\hline Germinação & $-0,2395 \mathrm{~ns}$ \\
Primeira Contagem & $0,0989 \mathrm{~ns}$ \\
Teste de Frio & $-0,0027 \mathrm{~ns}$ \\
Envelhecimento Acelerado & $-0,1345 \mathrm{~ns}$ \\
Condutividade Elétrica & $-0,1823 \mathrm{~ns}$ \\
\hline
\end{tabular}

ns $=$ não significado

Os testes de vigor de primeira contagem, de frio em rolo de papel sem solo, de envelhecimento acelerado, de condutividade elétrica e de emergência em campo indicaram diferenciação de qualidade entre lotes, destacando-se os lotes 3 e 5 com mais alto e baixo vigor, respectivamente (Tabela 1). O teste de frio é considerado pela ISTA (1981) e AOSA (1983) como um dos mais importantes na avaliação da qualidade de sementes. Segundo GRABE (1976), os lotes de qualidade adequada devem apresentar, no 
mínimo, 70 a $85 \%$ de plântulas normais como resultado do teste de frio. Este fato foi constatado em todos os lotes empregados neste estudo.

O teste de estresse hídrico (Tabela 3) apresentou resultados diferentes, em função do potencial hídrico a que as sementes foram expostas durante o período de germinação, onde se observou que, à medida que diminuiu o potencial hídrico, houve redução no comprimento das plântulas. Esta relação verificada, entre o potencial hídrico e comprimento das plântulas, concordam com os resultados de EMMERICH \& HARDEGREE (1991) e GERMU \& NAYLOR (1991). O maior crescimento das plântulas foi observado no potencial hídrico de $0 \mathrm{MPa}$; havendo redução progressiva com o decréscimo para -0,3 $\mathrm{MPa},-0,6 \mathrm{MPa}$ e -0,9 $\mathrm{MPa}$. Segundo ROGAN \& SIMON (1975) esta redução no crescimento das plântulas está associada ao estresse hídrico, que por sua vez atrasa a divisão e a elongação celular. As correlações entre o teste de estresse hídrico a $-0,3,-0,6$ e $-0,9 \mathrm{MPa}$ e a emergência em campo foram significativas, destacando-se o potencial hídrico de -0,6 MPa com correlação mais elevada (Tabela 4).

Tabela 3 - Valores médios do comprimento total de plântulas (mm) do teste de estresse híbrido, em seis lotes de sementes de sorgo (Sorghum bicolor (L.) Moench).

\begin{tabular}{ccccc}
\hline Lote & 0 & $-0,3$ & $-0,6$ & $-0,9$ \\
\hline 1 & $198,13 \mathrm{ab} *$ & $64,87 \mathrm{bc}$ & $45,75 \mathrm{a}$ & $18,87 \mathrm{a}$ \\
2 & $215,13 \mathrm{a}$ & $85,75 \mathrm{a}$ & $47,75 \mathrm{a}$ & $13,62 \mathrm{~b}$ \\
3 & $199,75 \mathrm{ab}$ & $72,37 \mathrm{ab}$ & $49,50 \mathrm{a}$ & $12,37 \mathrm{~b}$ \\
4 & $204,88 \mathrm{ab}$ & $49,25 \mathrm{c}$ & $33,75 \mathrm{~b}$ & $14,87 \mathrm{~b}$ \\
5 & $121,50 \mathrm{c}$ & $51,63 \mathrm{c}$ & $13,87 \mathrm{~d}$ & $8,50 \mathrm{c}$ \\
6 & $180,00 \mathrm{~b}$ & $74,50 \mathrm{ab}$ & $18,63 \mathrm{c}$ & $9,87 \mathrm{c}$ \\
\hline C V $(\%)$ & 11,33 & 17,63 & 14,85 & 14,28 \\
\hline
\end{tabular}

*Tratamento com médias não ligadas por mesma letra diferem pelo teste de Duncan em nível de $1 \%$ de erro.

O potencial de estresse hídrico sob -0,6 $\mathrm{MPa}$ apresentou correlação significativa em relação aos testes de germinação, primeira contagem, frio em rolo de papel sem solo, envelhecimento acelerado e emergência em campo (Tabela 5). Nos quatro ambientes de hidratação, o lote 5 apresentou o menor comprimento de plântulas (Tabela 3), demonstrando comportamento semelhante ao encontrado nos demais testes de vigor (Tabela 1).
Tabela 4 - Coeficientes de correlação simples (r) entre os resultados do teste de estresse híbrido conduzido em laboratório e a emergência em campo, em sementes de sorgo (Sorghum bicolor (L.) Moench).

\begin{tabular}{lc}
\hline Testes de laboratório x emergência em campo & $\mathrm{r}$ \\
\hline & \\
Estresse híbrido sob $0 \mathrm{MPa}$ & $-0,0277 \mathrm{~ns}$ \\
Estresse híbrido sob -0,3 MPa & $0,4047 *$ \\
Estresse híbrido sob -0,6 MPa & $0,6584^{* *}$ \\
Estresse híbrido sob -0,9 MPa & $0,4692^{*}$ \\
\end{tabular}

ns = não significado;

* = significado ao nível de $5 \%$ de erro

** = significado ao nível de $1 \%$ de erro;

Tabela 5 - Coeficientes de correlação simples (r) entre os resultados do teste de laboratório e de campo e o teste de estresses híbrido (-0,06 MPa).

\begin{tabular}{lc} 
Testes x teste de estresse híbrido (-0,6 MPa) & $\mathrm{r}$ \\
\hline Germinação & $0,5119^{*}$ \\
Primeira Contagem & $0,5910^{* *}$ \\
Frio & $0,5853^{* *}$ \\
Envelhecimento acelerado & $0,6111^{* *}$ \\
Condutividade elétrica & $-0,2217 \mathrm{~ns}$ \\
Emergência em campo & $0,6584^{* *}$ \\
\hline
\end{tabular}

ns = não significado

* = siginificado ao nível de $5 \%$ de erro;

** = significado ao nível de $1 \%$ de erro;

De acordo com MATTEWS \& POWELL (1986) e SÁ (1987), sementes de alto vigor são menos afetadas pelo estresse hídrico do que as de baixo vigor; fato observado neste estudo, para os potenciais de 0 , - 0,3 e -0,6 MPa, ao ser avaliado o crescimento das plântulas (Tabela 3). No potencial hídrico a -0,9 $\mathrm{MPa}$, o comportamento das sementes não obedeceu, de maneira geral, à ordem observada nos demais testes de vigor (Tabela 3), talvez devido o nível de estresse Ter sido bastante forte para as sementes, durante a germinação. 


\section{CONCLUSÃO}

O teste de germinação sob estresse hídrico de -0,6 MPa é indicado para avaliar a qualidade fisiológica de sementes de sorgo em situações desfavoráveis de disponibilidade hídrica no solo. Apresenta alta correlação com a emergência das plântulas em campo e com o teste de envelhecimento acelerado.

\section{REFERENCIAS BIBLIOGRÁFICAS}

ABDUL-BAKI, A.A., ANDERSON, J.D. Physiological and biochemical deterioration of seeds. In: KOZLOWSKI, T.T. (Ed.). Seed Biology. New York.: Aeademic Press, 1972. v. 2, p. $283-315$

ASSOCIATION OF OFFICIAL SEED ANALYSTS. Seed Vigor Test Committee. Seed vigor testing handbook. Lincoln, 1983. 88 p. (Contribution, 32).

BRASIL. Ministério da Agricultura e Reforma Agrária. Regras para análise de sementes: Brasília, 1992. 365 p.

CÍCERO, S.M., VIEIRA, R.D. Teste de frio. In: VIEIRA, R.D., CARVALHO, N.M. Testes de vigor em sementes. Jaboticabal: UNESP/FCAV, 1992. p. 58-62.

DELOUCHE, J.C., BASKJN, C.C. Acelerated aging techniques for predicting the relative storability ofseed lots. Seed Science and Technology, Zurich, v. 1, n. 2, p. 427-552, 1973.

El-SHARKAWI, HM, SPRINGUEL, 1. Germination ofsome crop seeds under reduced water potencial. Seed Science and Technology, Zurich, v. 5, p. 677-688, 1977.

EMMERICH, W.E., HARDEGREE, S.P. Poliethylene glycol solution contact effects on seed germination. Agronomy Journal, Madison, v. 82, p. 1103-1107, 1990.

EMMERICH, W.E., HARDEGREE, S.P. Seed germination in poliethylene glycol solution: effects offilter paper exclusion and water vapor loss. Crop Science, Madison, v. 31, p. 454458,1991 .

GERMU, M., NAYLOR, R.E.L. Effeets ofiow water availability - an germination of two sorghum eultivars. Seed Science and Technology, Zurieh, v. 19, p. 373-383, 1991.

GRABE, D.F. Measurement of seed vigor. Journal of Seed Technology, Springfield, v. 1, n. 2, p. 18-31, 1976.
HADAS, A.A. A suggested method for testing vigor under water stress in simulated arid conditions. Seed Science and Technology, Zurich, v. 5, p. 519-525, 1977.

INERNACIONAL SEED TESTING ASSOCIATION. Handbook ofvigour test methods. Zurich, 1981. $72 \mathrm{p}$

JOHNSON, R.R., WAX, L.M. Relationship of soybean germination and vigor tests to field performance. Agronomy Journal, Madison, v. 70, n. 2, p. 273-278, 1978.

MARCOS FILHO, J. Teste de envelhecimento acelerado. In: VIEIRA, R.D. \& CARVALHO N.M. Testes de vigor em sementes. Jaboticabal: FUNEP, 1994. p. 133-149.

MATTHEWS, S., POWELL, A.A. Environmental and physiological constraints on field performance of seeds Hortscience, Alexandria, v. 21, n. 5, p. 1125-1128, 1986.

MICHEL, B.E., KAUFMANN, M.R. The osmotic potencial of poliethylene glyeol 6.000. Plant Physiology, Rockville, v 51. p.914-916, 1973,

NAKAGAWA, J. Testes de vigor baseados na avaliação das plântulas. In: VIEIRA, R.D., CARVALHO, N.M. Testes de vigor em sementes. Jaboticabal: FUNEP. 1994. p. 49-85

PARMAR, M.T., MOORE, R.P. Carbowax 600. manitol, and sodium ehioride for simulation drought conditions in germination studies ofcom (Zea mays L.) ofstrong and weak vigor. Agronomy Journal, Madison, v. 60, p. 192-195, 1968.

PERRY, D.A. Introduction; methodology and appiication of vigor test; seediing growth and evaluation test. In: PERRY. D.A. (Ed.). Handbook of vigor tests methods. Zurich: Intemational Seed Testing Association, 1981. p. 3-20.

ROGAN, P.G., SIMON, E.W. Root growth and onset ofmitosis in germination Vicia faba. New Phytologist, London, v. 74, p. 263-265, 1975 .

SÁ, M.E. Relações entre qualidades fisiológica, disponibilidade hídrica e desempenho de sementes de soja (Glycine $\max$ (L.) Merril). Piracicaba, SP, 147 p. Tese (Doutorado em Fitotecnia), Escola Superior de Agricultura "Luiz de Queiroz", Universidade de São Paulo, 1987.

VIEIRA, R.D. Teste de condutividade elétrica. In: VIEIRA. R.D. CARVALHO, N.M. Testes de vigor em sementes. Jaboticabal: FUNEP, 1994. p. 103-132.

VILLELA, F.A., DONI FILHO, L., SIQUEIRA, E.L. Tabela de potencial osmótico em função da concentração de polietileno glicol 6.000 e da temperatura. Pesquisa Agro-pecuária Brasileira, Brasília, v. 26, n. 11/12, p. 1957-1968, 1991.

YAKLICH, R.W., KULIK, M.M. Evaluation of vigor tests in soybean seeds: relationship os standard germination test. Vigor classification, seediing lenth and tetrazolium staining, to field performance. Crop Science, Madison, v. 19, p.247252, 1979. 